\title{
FUNDAMENTAL DIMENSION AND SUSPENSION OF APPROXIMATE FIBRATIONS
}

\author{
P. F. DUVALL ${ }^{1}$ AND L. S. HUSCH
}

\begin{abstract}
Given an approximate fibration $f: X \rightarrow Y$ between ANR's a new approximate fibration $S f$ is constructed with the property that the fibers of $S f$ are the suspensions of the fibers of $f$. This construction is used together with an approximation theorem of T. Chapman to give conditions under which $\operatorname{Fd} X=$ $\mathrm{Fd} Y+\mathrm{Fd} f^{-1}(x)$.
\end{abstract}

1. Introduction. The proper map $f: X \rightarrow Y$ between locally compact ANR's is an approximate fibration if whenever $Z$ is a space and $\tilde{h}: Z \times\{0\} \rightarrow X$ and $H$ : $Z \times I \rightarrow Y$ are maps such that $f \tilde{h}=H \mid Z \times\{0\}$ and $\varepsilon$ is an open cover of $Y$, there is an extension $\tilde{H}$ of $\tilde{h}$ to all of $Z \times I$ such that $f \tilde{H}$ and $H$ are $\varepsilon$-close. If $Y$ is path connected, the point inverses of $F$ all have the same shape [CD1]. If $F$ has the shape of $f^{-1}(x)$, we say that $f$ is an approximate fibration with fiber $F$.

In this paper, we study the relation among the fundamental dimensions of $F, X$, and $Y$ for an approximate fibration $f$ with fiber $F$. In \$2, we introduce a device for producing an approximate fibration $S^{n} f$ whose fiber is the $n$-fold suspension of $F$ and use an approximation theorem of T. Chapman to show that if $n$ is sufficiently large, the algebraic topology of $S^{n} f$ is essentially that of a locally trivial bundle. $\$ 3$ is devoted to some algebraic preliminaries, and $\$ 4$ is devoted to proving

THEOREM 6. Suppose $f: X \rightarrow Y$ is an approximate fibration with fiber $F$, with $X, Y$ continua and with $\breve{\Pi}_{1}(F) \cong \check{\Pi}_{1}(Y) \cong 0$. Then $\mathrm{Fd} X=\mathrm{Fd} Y+\mathrm{Fd} F$ provided that $\breve{H}^{k}(F)$ is torsion free, where $k>0$ is the fundamental dimension of $F$.

We use $\check{\Pi}_{i}(X)$ to denote the $i$ th shape group [B2] of a space $X$. Cohomology is with $\mathbf{Z}$ ( $=$ integer) coefficients if no coefficient group is indicated. $\operatorname{Fd}(X)$ denotes the fundamental dimension of $X$, i.e., the least integer $n$ such that $X$ has the shape of an $n$-dimensional compactum. Our main tool for computing fundamental dimension is Nowak's theorem [N] that if $X$ is an approximately 1-connected compactum (this is equivalent to $\breve{\Pi}_{1}(X)=0$ when $X$ is a pointed FANR) and $\operatorname{Fd} X<\infty$, then $\operatorname{Fd} X=c(X)$, were $c(X)=\max \left\{n \mid \check{H}^{n}(X) \not \equiv 0\right.$. The symbol " $\cong$ is used to denote isomorphism, homeomorphism, or shape equivalence, its exact meaning will either be clear from the context or be irrelevant.

Received by the editors June 13, 1978 and, in revised form, April 24, 1979.

AMS (MOS) subject classifications (1970). Primary 55F65.

Key words and phrases. Approximate fibration, fundamental dimension, cohomology dimension.

${ }^{1}$ Research supported in part by an N.S.F. grant. 
2. Suspending the fibers of an approximate fibration. Let $f: X \rightarrow Y$ be a proper surjective map between locally compact separable metric ANR's. Let $Q$ be the Hilbert cube and 1: $Q \rightarrow Q$ be the identity map. The following is easily proved [CD1].

Proposition 1. $f: X \rightarrow Y$ is an approximate fibration if and only if $f \times 1$ : $X \times Q \rightarrow Y \times Q$ is an approximate fibration.

Let $S X$ be the space obtained by identifying two copies of the mapping cylinder $M(f)$ along their common top $X$. Formally, $S X$ is the quotient space $(X \times[-1,1] \amalg Y \times\{-1,1\}) / \sim$, where $\sim$ is the equivalence relation generated by $(x, i) \sim(f(x), i), i= \pm 1$. Define $S f: S X \rightarrow Y$ by $S f([x, t])=f(x)$. Note that for $y \in Y, S f^{-1}(y)$ is homeomorphic with the suspension $\Sigma f^{-1}(y)$ of $f^{-1}(y)$. We define $S^{n} X, S^{n} f$ by iterating this construction. By [B1, p. 116], $S X$ and, hence, $S^{n} X$ is a locally compact ANR for each $n$.

LEMMA 2. Let $f: X \rightarrow Y$ be an approximate fibration between locally compact ANR's with connected fiber. Then $S f$ is an approximate fibration.

Proof. By Proposition 1, it suffices to show that $S f \times 1: S X \times Q \rightarrow Y \times Q$ is an approximate fibration and by [CD2] it suffices to show that $S f \times 1$ is $k$-movable for all $k$. By Edwards [E], $Y \times Q$ is a $Q$-manifold, hence, each point in $Y \times Q$ has a neighborhood base of contractible open sets.

We now claim that it suffices to show:

If $U \supseteq V$ are contractible open sets in $Y \times Q$,

$\Pi_{i}\left((S f \times 1)^{-1}(U),(S f \times 1)^{-1}(V)\right) \cong 0$ for each $i$ and for

each base point in $(S f \times 1)^{-1}(V) .[S f \times 1:(S X) \times Q \rightarrow Y \times Q]$.

Proof of Claim. Since each fiber of $f$ is a pointed FANR [CD1], each fiber of $S f$ (and, hence, of $S f \times 1$ ) is also a pointed FANR [DNS].

Let $b \in Y \times Q$ and let $\left\{U_{i}\right\}_{i=1}^{\infty}$ be a sequence of compact ANR neighborhoods of $F_{b}=(S f \times 1)^{-1}(b)$ in $S X \times Q$ such that $U_{i+1} \subseteq$ int $U_{i}$ for all $i$ and $\cap_{i=1}^{\infty} U_{i}=$ $F_{b}$. Fix $k>0$. Since $F_{b}$ is a pointed FANR, by choosing a subsequence, if necessary, we may assume that $e_{i^{*}}$ image $e_{i+1^{*}}$ : image $e_{i+1^{*}} \rightarrow$ image $e_{i^{*}}$ is an isomorphism for each $i$ where $e_{i^{*}}$ is the homomorphism induced by inclusion $\Pi_{j}\left(U_{i}\right) \rightarrow \Pi_{j}\left(U_{i-1}\right)$ for $1 \leqslant j \leqslant k+1$. In addition, the natural projection $\check{\Pi}_{j}\left(F_{b}\right)=$ $\lim _{\leftarrow} \Pi_{j}\left(U_{i}\right) \rightarrow \Pi_{j}\left(U_{i}\right)$ induces an isomorphism $\check{\Pi}_{j}\left(F_{b}\right) \rightarrow$ image $e_{i+1}$ for all $i$ and $1 \leqslant j \leqslant k+1$.

Let $W$ be a contractible open set in $Y \times Q$ containing $b$ such that for some $i$, $(S f \times 1)^{-1}(W) \supseteq U_{i}$ and choose a contractible open neighborhood $W_{1}$ of $b$ in $Y \times Q$ such that $U_{i} \supseteq(S f \times 1)^{-1}\left(W_{1}\right)$. By using (*) and some algebra, it is easy to show that the composition $\check{\Pi}_{j}\left(F_{b}\right) \rightarrow \Pi_{j}\left(U_{i}\right) \rightarrow \Pi_{j}\left((S f \times 1)^{-1}(W)\right)$ is an isomorphism for $j<k$ and an epimorphism for $j=k+1$.

Recall that to show that $S f \times 1$ is $k$-movable, we must show that for each $c \in Y \times Q$ and each neighborhood $U_{0}$ of the fiber $F_{c}$, there are neighborhoods $U$ and $V$ of $F_{c}$ such that $V \subseteq U \subseteq U_{0}$ and $\check{\Pi}_{1}\left(F_{b}\right)$ is realized as the image of $\Pi_{i}(V)$ in 
$\Pi_{i}(U), 0 \leqslant i \leqslant k$, for every fiber $F_{b} \subseteq V$. Given $c$ and $U_{0}$, choose a contractible open set $W$ containing $c$ in $Y \times Q$ such that $(S f \times 1)^{-1}(W) \subseteq U_{0}$. Let $U=V=$ $(S f \times 1)^{-1}(W)$. For each $b$ such that $F_{b} \subseteq V, \check{\Pi}_{j}\left(F_{b}\right) \rightarrow \Pi_{j}(V)$ is an isomorphism for $j<k$. Hence $S f \times 1$ is $k$-movable and the claim is proved. Now we proceed to prove (*).

If $A \subseteq S X \times Q$, define $A_{+}=\{([x, t], q) \in A \mid t \geqslant 0\}, A_{-}=\{([x, t], q) \in A \mid t$ $<0\}, A_{0}=A_{+} \cap A_{-}$. Now let $U \supseteq V$ be as in (*). If $y \in V$, the exact sequence from [CD1] gives the following commutative diagram where the vertical arrows are inclusion induced.

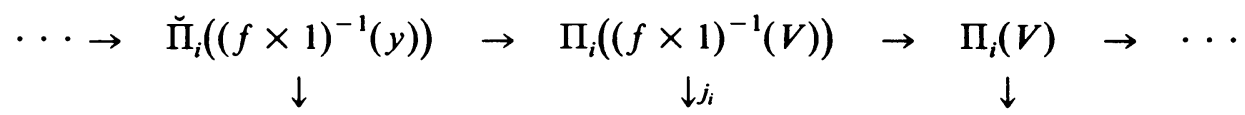

$$
\begin{aligned}
& \cdots \rightarrow \check{\Pi}_{i}\left((f \times 1)^{-1}(y)\right) \rightarrow \Pi_{i}\left((f \times 1)^{-1}(U)\right) \rightarrow \Pi_{i}(U) \rightarrow \cdots
\end{aligned}
$$

Since $U$ and $V$ are contractible, it follows that $j_{i}$ is an isomorphism for each $i$, so that $\Pi_{*}\left((f \times 1)^{-1}(U),(f \times 1)^{-1}(V)\right) \cong 0$. By the Hurewicz theorem,

$$
H_{*}\left((f \times 1)^{-1}(U),(f \times 1)^{-1}(V)\right) \cong 0 .
$$

Now

$$
H_{*}\left((f \times 1)^{-1}(U),(f \times 1)^{-1}(V)\right) \cong H_{*}\left((S f \times 1)^{-1}(U)_{0},(S f \times 1)^{-1}(V)_{0}\right) \cong 0
$$

and

$$
H_{*}\left((S f \times 1)^{-1}(U)_{ \pm},(S f \times 1)^{-1}(V)_{ \pm}\right) \cong H_{*}(U, V) \cong 0
$$

so that $H_{*}\left((S f \times 1)^{-1}(U),(S f \times 1)^{-1}(V)\right) \cong 0$ by the Mayer-Vietoris sequence. Since $\Pi_{1}\left((S f \times 1)^{-1}(U)\right) \cong 0$ by the Van Kampen theorem, we can apply the relative Hurewicz theorem to conclude that $\Pi_{*}\left((S f \times 1)^{-1}(U),(S f \times 1)^{-1}(V)\right) \simeq$ 0.

Theorem 3. Suppose that $p: X \rightarrow Y$ is an approximate fibration between locally compact ANR's with connected fiber $F$. Suppose that $Y$ has the homotopy type of an $n$-complex, $n<\infty$. Then there is a locally trivial fibration $f: S^{n+1} X \times Q \rightarrow Y \times Q$ with fiber $K$ such that

(i) $\operatorname{Sh} K=\operatorname{Sh}\left(\Sigma^{n+1} F\right)$,

(ii) there is a homotopy equivalence $\phi$ of $S^{n+1} X \times Q$ onto itself making

$$
\begin{array}{ccc}
S^{n+1} X \times Q & \stackrel{\phi}{\rightarrow} & S^{n+1} X \times Q \\
S^{n+1} p \times 1 \searrow & & \swarrow f \\
& Y \times Q &
\end{array}
$$

commute.

Proof. By Lemma $2, S^{n+1} p \times 1$ is an approximate fibration with $n$-shape connected fibers. Thus by [C, Theorem 2], $S^{n+1} p \times 1$ is homotopic to (can be approximated by) a locally trivial map. Conclusions (i) and (ii) follow by applying the proof of Theorem 1.2 of [F] with $g=$ id. 


\section{Algebraic lemmas.}

Lemma 4. Let $f: X \rightarrow Y$ be an Hurewicz fibration with connected fiber $F$, where $X$ and $Y$ are compact connected $A N R$ 's and

(a) $\breve{\Pi}_{1}(F) \cong \Pi_{1}(Y) \cong 0$,

(b) $\operatorname{Fd}(F)=k<\infty, \operatorname{Fd}(Y)=l<\infty$, and

(c) $H^{k}(F)$ is torsion free.

Then $\operatorname{Fd}(X)=k+l$.

Proof. It follows from the exact sequence of a fibration that $\Pi_{1}(X) \simeq 0$, so by [N1], it suffices to show that $H^{k+l}(X) \neq 0$ and $H^{n}(X) \simeq 0$ if $n>k+l$. The conclusion follows from a standard spectral sequence argument, which we sketch for completeness. We use the notation of [S]. In the cohomology spectral sequence of $f$, we have $E_{2}^{* p, q} \cong H^{p}\left(Y ; H^{q}(F)\right)$, so that $E_{r}^{* p, q} \cong E_{\infty}^{* p, q} \cong 0$ if $p>l$ or $q>k$, and in particular, if $p+q=k+l, E_{\infty}^{* p, q} \cong 0$ unless $p=l$ and $q=k$. Now $E_{2}^{* l, k} \cong A=H^{l}\left(Y, H^{k}(F)\right)$ and $A \neq 0$ by the universal coefficient theorem, since $H^{l}(Y) \neq 0$ and $H^{k}(F)$ is torsion free.

Suppose that we have shown that for some $r \geq 2, E_{r}^{* l, k} \simeq A$. Then $E_{r+1}^{* l, k}$ is kernel mod image in

$$
E_{r}^{* l-r, k+r-1} \rightarrow A \rightarrow E_{r}^{* l+r, k-r+1}
$$

Since the two extreme groups are zero, it follows that $E_{r+1}^{* l, k} \simeq E_{\infty}^{* l, k} \simeq A$.

Now $H^{k+l}(X)$ has a filtration

$$
0=D^{* k+l+1,-1} \subset D^{* k+l, 0} \subset \cdots \subset D^{* k+l-i, i} \subset \cdots \subset D^{* 0, k+l} \simeq H^{k+l}(X),
$$

where each $D^{* k+l-i,+i} / D^{* k+l-i+1, i-1} \cong E_{\infty}^{* k+l-i, i}$. It follows that $H^{k+l}(X) \cong A$ $\neq 0$. A similar argument shows that $H^{n}(X) \cong 0$ if $n>k+l$.

REMARKs. If $X$ and $Y$ are merely assumed to be locally compact, then our argument shows that Lemma 4 remains true if " $F d "$ is replaced by " $c$ " throughout the statement.

This lemma is surely known in its cohomological form by algebraic topologists; compare, for example [S, Proposition 3, p. 466] and [R, Lemma 6.1].

LEMMA 5. Suppose $p: X \rightarrow Y$ is an approximate fibration between locally compact $A N R$ 's with connected fiber $F, c(F)=k>0$. If $c(X)=m>c(Y)$, then (a) $c(\Sigma F)$ $=k+1$ and $(\mathrm{b}) c(S X)=m+1$.

Proof. (a) is immediate. For (b), note that the Mayor-Vietoris sequence of $\left(S X ; S_{+} X, S_{-} X\right)$ gives an exact sequence

$$
\cdots \leftarrow H^{i+1}(Y)+H^{i+1}(Y) \leftarrow H^{i+1}(S X) \leftarrow H^{i}(X) \leftarrow H^{i}(Y)+H^{i}(Y) \leftarrow \cdots
$$

so that $H^{i+1}(S X) \simeq H^{i}(X)$ if $i>c(Y)$.

\section{Proof of Theorem 6.}

Theorem 6. Suppose that $p: X \rightarrow Y$ is an approximate fibration with fiber $F$, where $X$ and $Y$ are compact connected $A N R$ 's with $\Pi_{1}(Y) \simeq \check{\Pi}_{1}(F) \simeq 0, \check{H}^{k}(F)$ torsion free, where $\operatorname{Fd}(F)=k>0$. Then $\mathrm{Fd} X=\mathrm{Fd} F+\mathrm{Fd} Y$. 
Proof. First, let us assume that $F$ is connected. Let Fd $X=m, \operatorname{Fd} Y=l$. Choose $N>\max (0, l-m)$ and let $\bar{X}=X \times T^{N}$ where $T^{N}$ is the $N$-torus, the product of $N$ copies of the 1-sphere, $\bar{p}: \bar{X} \rightarrow Y$ the composition $X \times$ $T^{N} \underset{\text { proj }}{\rightarrow} X \underset{p}{\rightarrow} Y$. Then $\bar{p}$ is an approximate fibration with fiber $\bar{F} \cong F \times T^{N}$ [CD1]. By the Künneth formula $c(\bar{F})=k+N, c(\bar{X})=m+N$ and $\check{H}^{k+N}(\bar{F})$ is torsion free. (In fact, $\breve{H}^{k+N}(F) \cong \breve{H}^{k}(F)$.) Now choose $n$ such that $Y$ has the homotopy type of an $(n-1)$-complex. By Theorem 3 , there is a Hurewicz fibration $f$ : $S^{n} \bar{X} \times Q \rightarrow Y \times Q$ whose fiber has the shape of $\Sigma^{n} \bar{F}$. By Lemma 4,

$$
\operatorname{Fd}\left(S^{n} \bar{X} \times Q\right)=\operatorname{Fd}\left(\Sigma^{n} \bar{F}\right)+\operatorname{Fd}(Y),
$$

hence, $c\left(S^{n} \bar{X} \times Q\right)=c\left(\Sigma^{n} \bar{F}\right)+c(Y)$. Lemma 5 gives that $c(\bar{X})+n=c(\bar{F})+n$ $+c(Y)$, so that we have

$$
\begin{gathered}
c(X)+N+n=c(F)+N+n+c(Y), \text { or } c(X)=c(F)+c(Y) \\
\text { Fd } X=\operatorname{Fd} F+\operatorname{Fd} Y, \text { by }[\mathrm{N1}] .
\end{gathered}
$$

Now suppose that $F$ is not connected. Let $p_{0}: X \rightarrow Y_{0}$ and $q: Y_{0} \rightarrow Y$ be a monotone and a light mapping, respectively, such that $q p_{0}=p$. It is easily shown that $p_{0}$ is an approximate fibration whose fiber is a component of $F$ and $q$ is a covering map. (It follows that the components of $F$ have the same shape.) Apply the first part of this proof.

REMARKs. In Theorem 6 and Lemma 4 some homological assumption is necessary, for there are simple examples of 1-connected polyhedra $X$ and $Y$ with $\mathrm{Fd}(X \times Y)<\mathrm{Fd} X+\mathrm{Fd} Y[\mathrm{~N}]$. Our argument actually shows that Theorem 6 and Lemma 4 remain true if the condition $\breve{H}^{k}(F)$ torsion free is replaced by (*) $H^{l}\left(Y ; \breve{H}^{k}(F)\right) \neq 0$. By tracing the role of (*) through the several lemmas, one can also show that (*) is necessary. As in the remarks after Lemma 4, Theorem 6 remains true for locally compact $X$ and $Y$ if " $F d$ " is replaced by " $c$ ".

\section{REFERENCES}

[B1] K. Borsuk, Theory of retracts, Polish Scientific Publishers, Warsaw, 1967.

[B2] __ Theory of shape, Polish Scientific Publishers, Warsaw, 1975.

[C] T. Chapman, Approximating maps into fiber bundles by homeomorphisms (to appear).

[CD1] D. Coram and P. Duvall, Approximate fibrations, Rocky Mountain J. Math. 7 (1977), 275-288.

[CD2] __, Approximate fibrations and a movability condition for maps, Pacific J. Math. 72 (1977), 41-56.

[DNS] J. Dydak, S. Nowak and M. Strok, On the union of two FANR-sets, Bull. Acad. Polon. Sci. Sér.

Sci. Math. Astronom. Phys. 24 (1976), 485-489.

[E] R. Edwards, A locally compact ANR is a Hilbert cube manifold factor (to appear).

[EG] D. Edwards and R. Geoghegan, Shapes of complexes, ends of manifolds, homotopy limits and the Wall obstruction, Ann. of Math. (2) 101 (1975), 521-535.

[F] S. Ferry, Approximate fibrations with nonfinite fibers, Proc. Amer. Math. Soc. 64 (1977), 335-345.

[N] S. Nowak, On the fundamental dimension of approximately 1-connected compacta, Fund. Math. 89 (1975), 61-79.

[R] F. Raymond, Local triviality for Hurewicz fiberings of manifolds, Topology 3 (1965), 43-57.

[S] J. P. Serre, Homologie singulière des espaces fibrés, Ann. of Math 54 (1951), 425-505.

Department of Mathematics, Oxlahoma State University, Stillwater, Okiahoma 74074

Department of Mathematics, University of Tennessee, Knoxville, Tennessee 37916 